\title{
The active electric sense of weakly electric fish: from electric organ discharge to sensory processing and behaviour
}

\author{
Rüdiger Krahe ${ }^{a}$ \\ Department of Biology, McGill University, Montreal, Quebec H3A 1B1, Canada
}

\begin{abstract}
Sensory systems have been shaped by evolution to extract information that is relevant for decision making. In order to understand the mechanisms used by sensory systems for filtering the incoming stream of sensory input, it is important to have a quantitative understanding of the natural sensory scenes that are to be processed. Weakly electric fish lead a rather cryptic nocturnal life in often turbid tropical rainforest streams. They produce electric discharges and sense perturbations of their selfgenerated electric field for prey detection and navigation, and also use their active sense for communication in the context of courtship and aggression. The fact that they produce their electric signals throughout day and night permits the use of electrode arrays to track the movements of multiple individual fish and monitor their communication interactions, thus offering a window into their electrosensory world. This approach yields unprecedented access to information on the biology of these fishes and also on the statistical properties of the sensory scenes that are to be processed by their electrosensory system. The electrosensory system shares many organizational features with other sensory systems, in particular, the use of multiple topographic maps. In fact, the sensory surface (the skin) is represented in three parallel maps in the hindbrain, with each map covering the receptor organ array with six different cell types that project to the next higher level of processing. Thus, the electroreceptive body surface is represented a total of 18 times in the hindbrain, with each representation having its specific filter properties and degree of response plasticity. Thus, the access to the sensory world of these fish as well as the manifold filtering of the sensory input makes these fish an excellent model system for exploring the cell-intrinsic and network characteristics underlying the extraction of behaviourally relevant sensory information.
\end{abstract}

\footnotetext{
${ }^{\mathrm{a}}$ Corresponding author: rudiger.krahe@,mcgill.ca
} 